\title{
A CRÉPINETTE SIGN
}

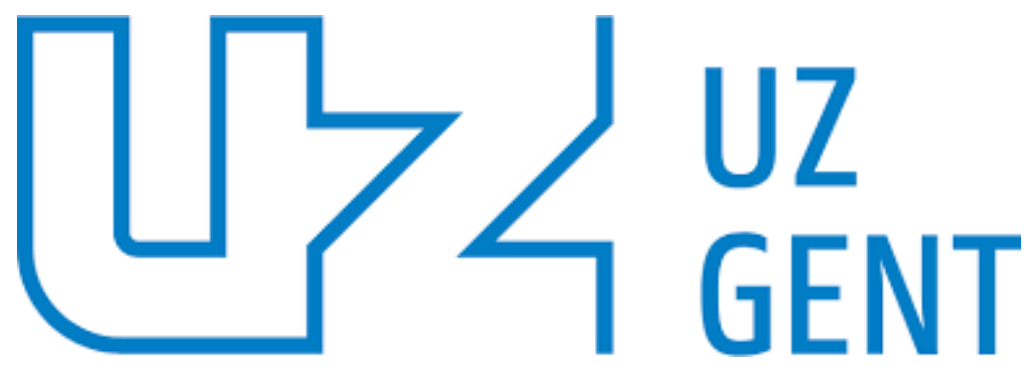

\section{S. Kennes ${ }^{1}$, C. De Vriendt ${ }^{1}$, D. Mazure ${ }^{1}$, P. De Visschere ${ }^{2}$, T. Kerre ${ }^{1}$ Department of Hematology ${ }^{1}$, Radiology2 ${ }^{2}$, Ghent University Hospital, Ghent, Belgium.}

\section{INTRODUCTION}

Extramedullary invasion of B-cell acute lymphoblastic leukemia (B-ALL) during cytomorphological remission (CR) has been frequently reported in the central nervous system [1]. Abdominal involvement in apparent remission is rare [2] and occurs mostly in case of leukemic relapse [3,4]

\section{CASE PRESENTATION}

A 58-year-old man known with B-ALL was hospitalized because of confusion after recent fall.

He underwent allogeneic stem cell transplantation with a CMV negative MUDdonor as consolidation after achieving CR2 with chemotherapy (GMALL07/2003) in 2016. Acute graft-versus-host disease of the skin and bowel was successfully treated with corticosteroids, but was followed by recurrent CMV disease of the gastrointestinal tract. Recent bone marrow puncture before admission showed CR with no arguments for MRD by means of flow cytometry.

Although he had a normal clinical and neurological examination, the patient was admitted for suspected meningo-encephalitis. Lumbar puncture and cranial CT scan were normal. The confusion could also be part of a systemic inflammatory response secondary to an infection without focus, considering the absence of pleocytosis. After initial improvement with ceftriaxone and aciclovir, antibiotics were switched to piperacillin-tazobactam because of rise in CRP on the $6^{\text {th }}$ day of admission. Because there was no evidence of herpes meningitis, aciclovir was discontinued and Valcyte maintenance was restarted. The latter was rapidly switched to Cymevene because of CMV reactivation and the antibiotic treatment was extended to Meropenem because of further rise in CRP. Ultrasonography, executed because of cholestasis parameters, showed right hydronephrosis and liver steatosis, the latter due to chronic systemical corticosteroid therapy. Subsequently, abdominal CT (figure 1) displayed extensive reticulo-nodular perirenal and -adrenal infiltration with encasement and invasion of the pyelum and ureter, besides nodular thickening with peritoneal and mesenterial invasion. Imaging showed similarities with crépinette, a gastronomic term for meat wrapped in abdominal membranes (figure 1).

Urgent laparoscopy revealed a diffuse metastatic process, confirmed on pathology. Flow cytometric analysis of the biopsy demonstrated a CD10/CD20/ CD34+ population, showing the original leukemia-associated phenotype (LAIP). Systemic corticosteroids were associated because of the systemic inflammation and malignant lactic acidosis in this invasive extramedullary B-ALL. At the request of the patient and his family, active treatment was stopped and palliative sedation was started. The patient unfortunately succumbed 11 days later.

\section{DISCUSSION and CONCLUSION}

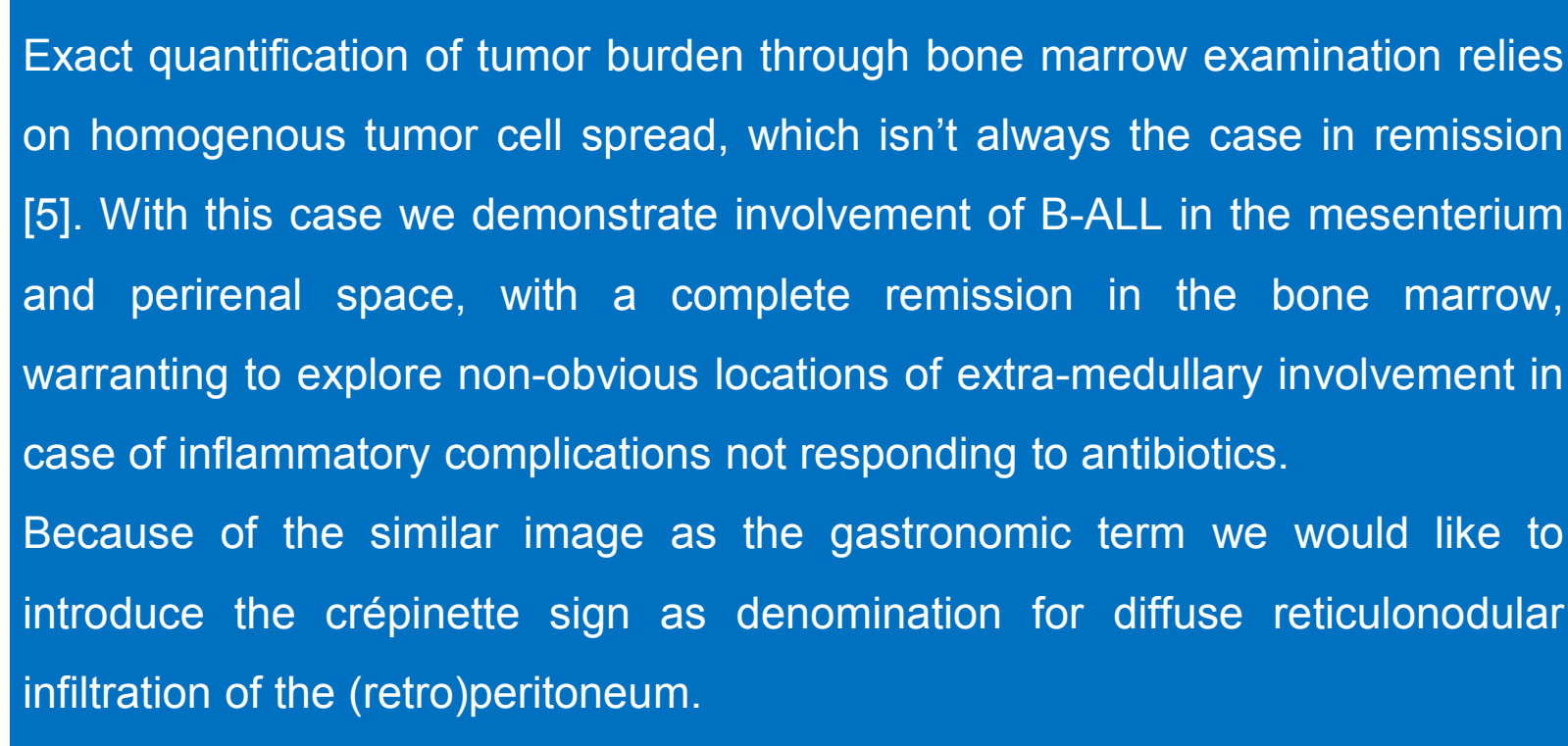

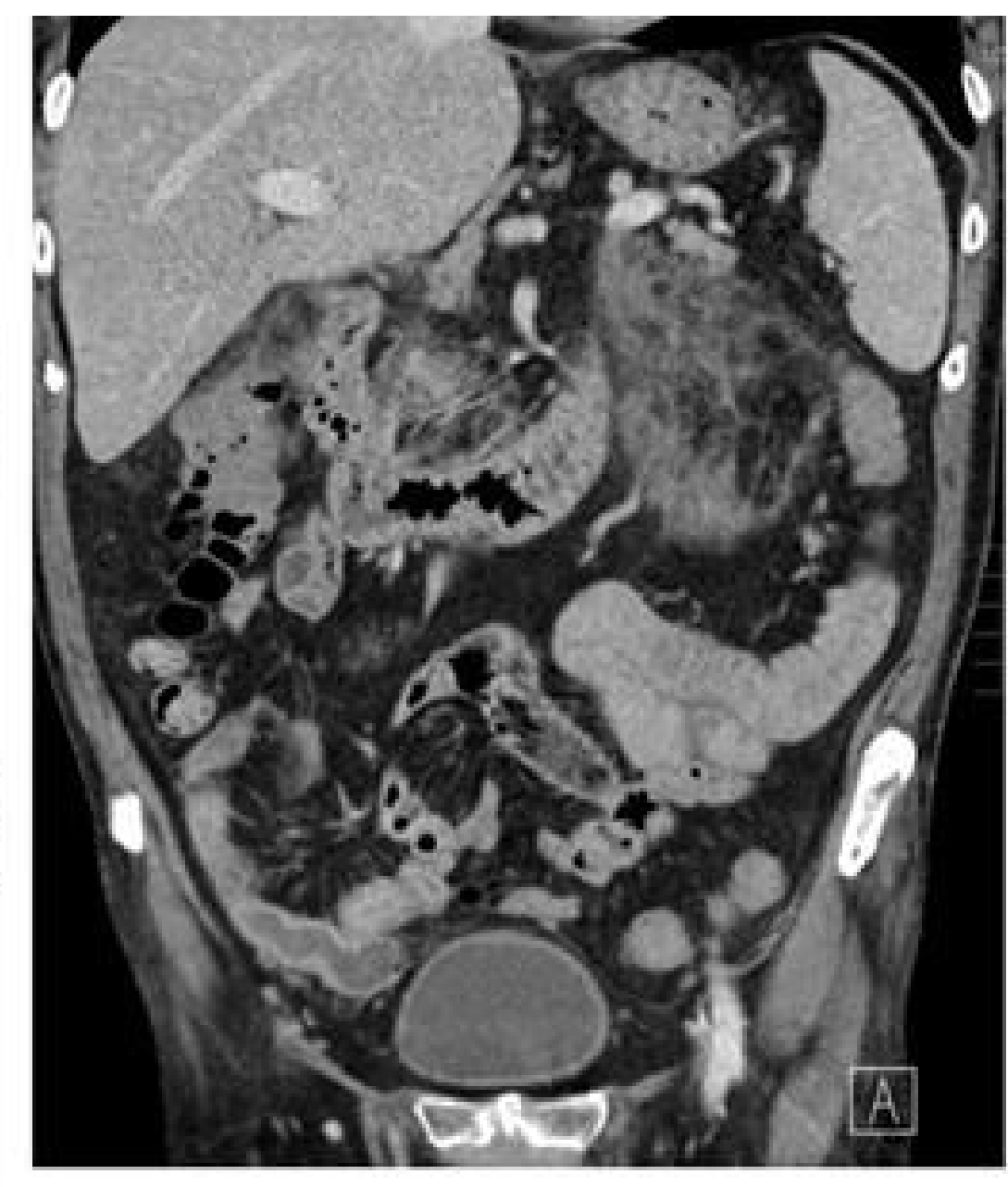

Figure 1: axial and coronal images of the excesive reticulonodular infiltration perirenal, (retro)peritoneal and mesenterial. Right under: crépinette (i.e. minced meat wrapped in abdominal membranes), a similar image.
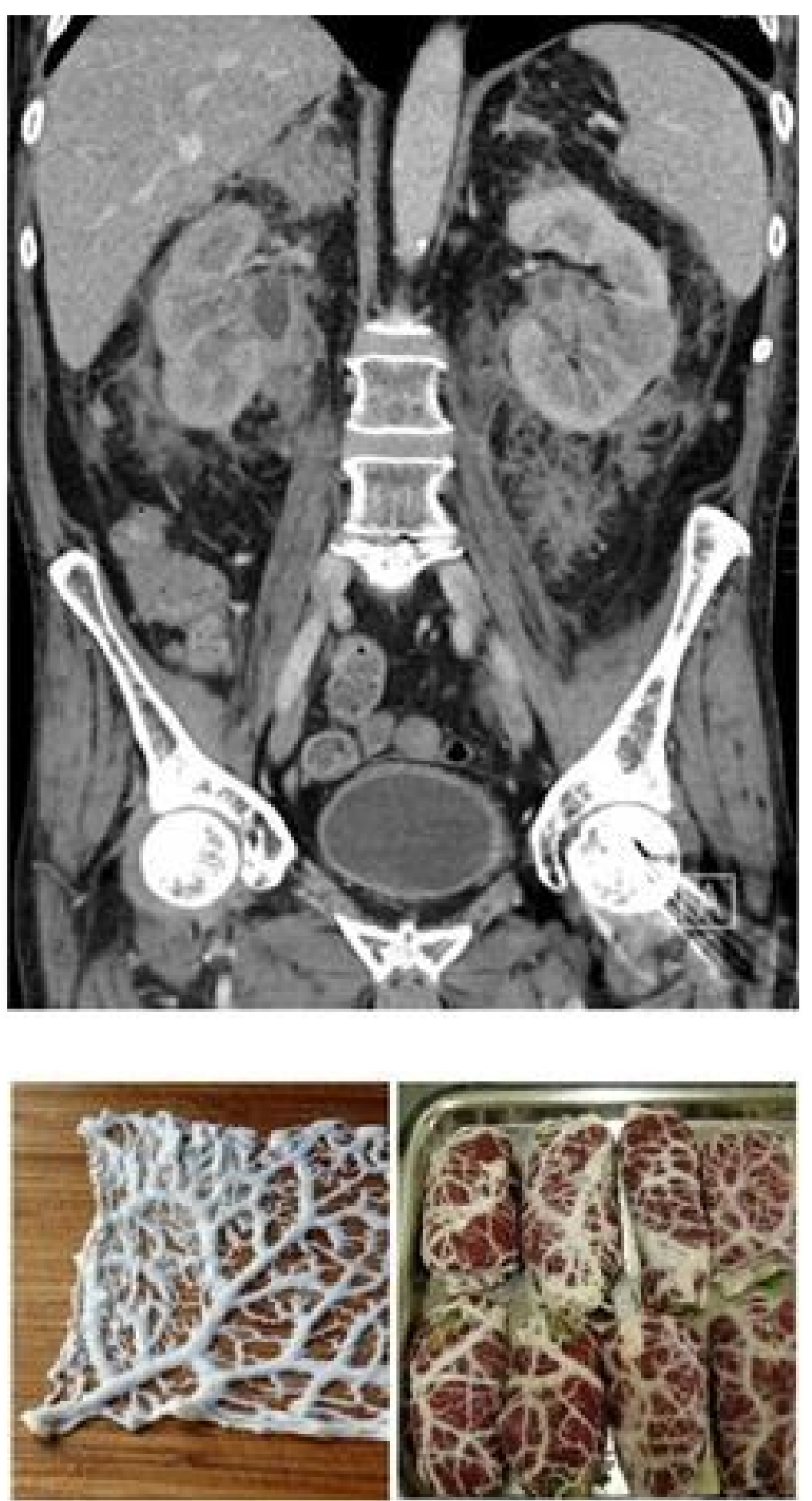

\section{REFERENCES}

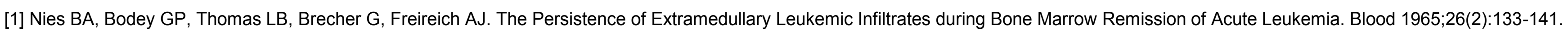
[2] Everett CR, Haggard ME, Levin WC. Extensive Leukemic Infiltration of the Gastrointestinal Tract during Apparent Remission in Acute Leukemia. Blood 1963;22(1):92-99.

[3] Ebert EC, Hagspiel KD. Gastrointestinal manifestation of leukemia. J. Gastroenterol. Hepatol. 2012;27(3):458-463.

[4] Cecalupo AJ, Frankel LS, Sullivan MP. Pelvic and ovarian extramedullary leukemic relapse in young girls. Cancer 1982;50(3):587-593.

[5] Buckley SA, Appelbaum FR, Walter RB. Prognostic and therapeutic implications of minimal residual disease at the time of transplantation in acute leukemia. Bone Marrow Transplantation 2013;48:630-641. 